\title{
Methyltransferase and demethylase profiling studies during brown adipocyte differentiation
}

\author{
Min Jeong Son ${ }^{1, \#}$, Won Kon Kim ${ }^{1,2, \#}$, Kyoung-Jin Oh ${ }^{1}$, Anna Park ${ }^{1}$, Da Som Lee ${ }^{1}$, Baek Soo Han ${ }^{1,2}$, Sang Chul Lee ${ }^{1,2, *}$ \\ $\mathcal{E}$ Kwang-Hee Bae ${ }^{1,2, *}$ \\ ${ }^{1}$ Metabolic Regulation Research Center, KRIBB, ${ }^{2}$ Department of Functional Genomics, University of Science and Technology (UST), \\ Daejeon 34141, Korea
}

\begin{abstract}
Although brown adipose tissue is important with regard to energy balance, the molecular mechanism of brown adipocyte differentiation has not been extensively studied. Specifically, regulation factors at the level of protein modification are largely unknown. In this study, we examine the changes in the expression level of enzymes which are involved in protein lysine methylation during brown adipocyte differentiation. Several enzymes, in this case SUV420H2, PRDM9, MLL3 and JHDM1D, were found to be up-regulated. On the other hand, Set7/9 was significantly down-regulated. In the case of $S U V 420 H 2$, the expression level increased sharply during brown adipocyte differentiation, whereas the expression of SUV42OH2 was marginally enhanced during the white adipocyte differentiation. The knock-down of SUV420H2 caused the suppression of brown adipocyte differentiation, as compared to a scrambled control. These results suggest that SUV420H2, a methyltransferase, is involved in brown adipocyte differentiation, and that the methylation of protein lysine is important in brown adipocyte differentiation. [BMB Reports 2016; 49(7): 388-393]
\end{abstract}

\section{INTRODUCTION}

White adipose tissue (WAT) stores extra energy as lipid droplets in white adipocyte cells. In contrast, brown adipose tissue (BAT) dissipates the extra energy as heat by uncoupling the respiratory chain of oxidative phosphorylation in mitochondria.

*Corresponding authors. Kwang-Hee Bae, Tel: +82-42-860-4268; Fax: +82-42-860-4269; E-mail: khbae@kribb.re.kr, Sang Chul Lee, Tel: +82-42-860-4145; Fax: +82-42-860-4593; E-mail: lesach@ kribb.re.kr

${ }^{\#}$ These two authors contributed equally.

http://dx.doi.org/10.5483/BMBRep.2016.49.7.062

Received 22 March 2016, Revised 30 March 2016, Accepted 2 May 2016

Keywords: Brown adipocytes, Demethylase, Methyltransferase, Obesity, SUV420H2
Energy expenditure through non-shivering thermogenesis in BAT serves either to maintain the body's temperature against cold exposure or to waste food energy, indicating its role in both thermal and energy balancing processes. Recently, a correlation between obesity and the amount of BAT in humans has been reported by many research groups (1-3). Thus, a deeper understanding of the molecular and cellular mechanisms of brown adipocyte differentiation is important in terms of the treatment and prevention of obesity. However, the molecular mechanisms and signal transduction of brown adipocyte differentiation have not been extensively studied, as compared to studies of white adipocyte differentiation $(4,5)$.

Post-translational modifications (PTMs) of proteins are common and typical regulatory mechanisms by which organisms control key cellular processes, such as the cell cycle, cell survival, and cell proliferation and differentiation (5). Specifically, the lysine methylation of histones is known to be important for the epigenetic regulation of transcription and chromatin in eukaryotes. Recently, it was reported that lysine methylation of non-histone proteins is also very crucial in various key cellular processes $(6,7)$. Furthermore, the cross-talk between lysine methylation and other PTMs has an effect on the functions of modified proteins (8). However, the relationship between lysine methylation and brown adipocyte differentiation has not been studied extensively $(9,10)$. The site and degree of lysine methylation is dynamically regulated by a balanced action between lysine methyltransferases (KMTs) and lysine demethylases (KDMs).

SUV420H2 (also known as KMT5C), together with SUV420H1, function as histone methyltransferases specifically trimethylating histone $\mathrm{H} 4$ on lysine-20 (H4K20) (11). Histone $\mathrm{H} 4 \mathrm{~K} 20$ trimethylation induces epigenetic transcriptional repression. In addition, SUV420H2 functions in pericentric heterochromatin regions, and thereby plays a key role in the establishment and maintenance of constitutive heterochromatin in these regions (12). SUV42OH-double-null mice are perinatally lethal and are bred to lose nearly all H4K2Ome3 and H4K20me2 states. Additionally, they show impaired genome instability and programmed DNA rearrangements (13). Recently, it was reported that the loss of H4K2Ome3 in tumor cells by the decreased expression of a H4K20-specific 
methyltransferase, SUV420H2, induced the expression of cancer-promoting genes, including tensin-3 (14).

In this study, we undertook profiling studies of KMTs and KDMs during brown adipocyte differentiation. In addition, given the results from a knock-down experiment on $\mathrm{SUV} 42 \mathrm{OH}$, we suggest that SUV420H2 is involved in brown adipocyte differentiation, and that lysine methylation plays an important role in this process as well. Brown preadipocyte differentiation

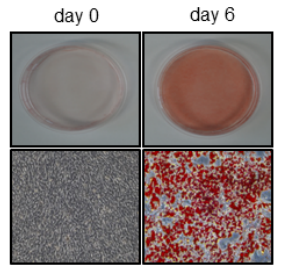

B.
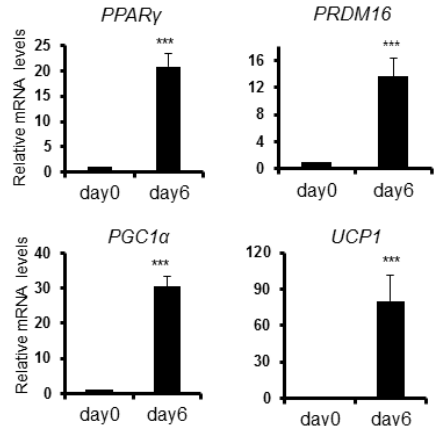

c.

A.

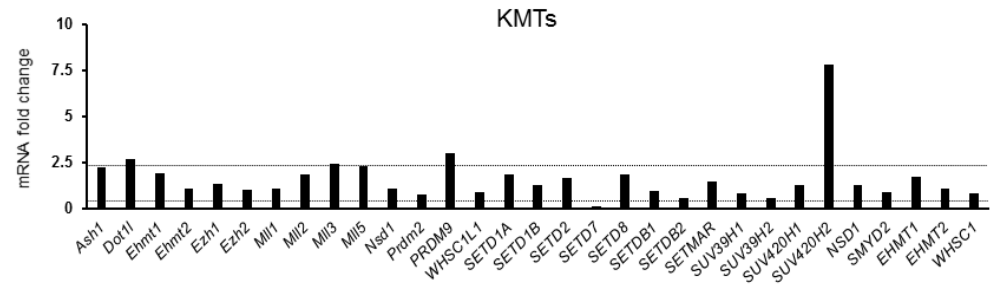

B.

c.

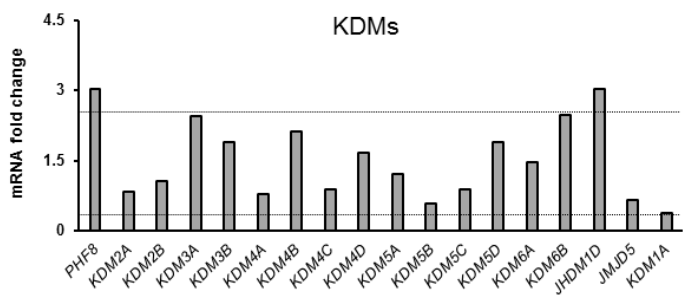

\section{RESULTS AND DISCUSSION}

First, immortalized brown preadipocytes were induced into mature brown adipocytes. As shown in Fig. 1A, the immortalized brown preadipocytes were successfully differentiated and stained by Oil-Red O. Additionally, the mRNA levels of several markers, in this case PPAR $\gamma, P G C 1 \alpha, P R D M 16$ and $U C P 1$, were dramatically increased during brown adipocyte differentiation (Fig. 1B). The protein levels of UCP1 and adipocyte protein 2 (aP2) were also significantly up-regulated

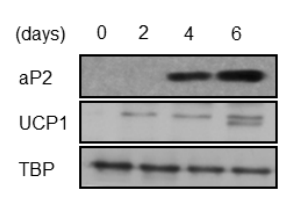

.
Fig. 2. KMT and KDM profiling analyses during brown adipocyte differentiation. (A) The expression levels of a total of 31 KMTs were examined by qPCR array analysis. (B) The expression levels of a total of 18 KMTs were examined by qPCR array analysis. (C) Positive markers of differentiation were also examined in mature brown adipocyte by qPCR. The enzymes showing significant differences $( \pm$ over three fold, $P$ $<0.05)$ were defined as differentially expressed. 
(Fig. 1C), indicating that efficient differentiation occurred under our experimental conditions. Next, to identify the KMTs and KDMs involved in brown adipocyte differentiation, we performed a qPCR array experiment of samples obtained from both preadipocytes and mature brown adipocytes, using immortalized brown preadipocyte cells. We analyzed the expression changes of 31 methyltransferases and 18 demethylases. Among these, several enzymes showed differential expression patterns during brown adipocyte differentiation (Fig. 2). In particular, SUV42OH2, PRDM9, MLL3, PHF8 and $J H D M 1 D$ were increased during brown adipocyte differentiation. On the other hand, the Set7/9 was significantly suppressed. In the cases of PRDM9 and JHDM1D, the changes of the expression levels were validated by real-time PCR (Fig. $3 \mathrm{~A})$. However, there was no significant detectable change in the case of PHF8 during differentiation. Among the KMTs or KDMs showing differential expression patterns during brown adipocyte differentiation, SUV42OH2 showed a dramatic expression change, but there have been no reports about its involvement in adipocyte differentiation or in the insulin signaling pathway. The changes at the level of both the mRNA and the protein were also assessed, showing that the mRNA and protein levels increase sharply during the late stages of brown adipocyte differentiation (Figs. 3B and 3C). This result suggests that the expression of $S U V 42 O H 2$ is important during the late stages of adipogenesis. During white adipocyte differentiation, the expression of SUV42OH2 showed a slight increase. However, the degree of the increase is significantly smaller than in the case of brown adipocyte differentiation. Next, we more closely investigated the effect of SUV42OH2 on

A.

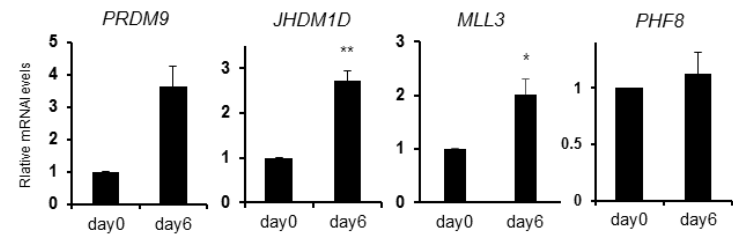

B.

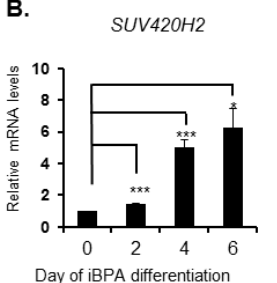

Day of iBPA differentiation

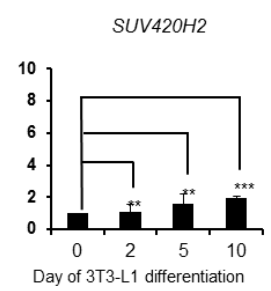

C.

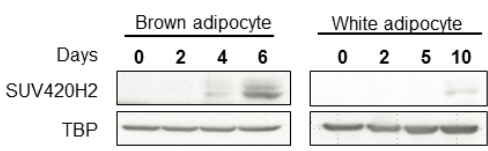

A.

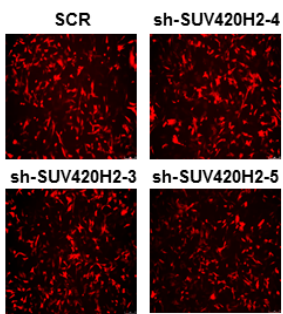

D.

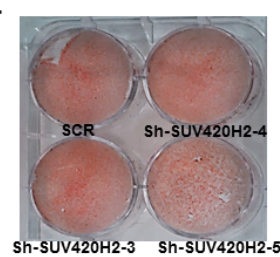

B.

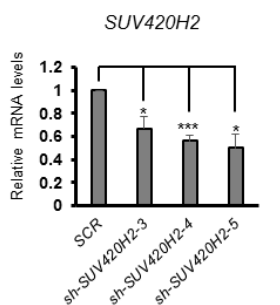

E.

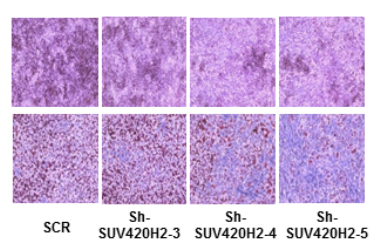

c.

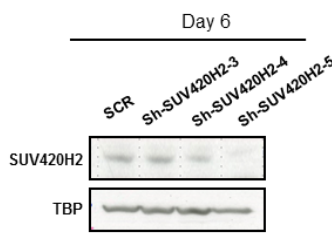

F.

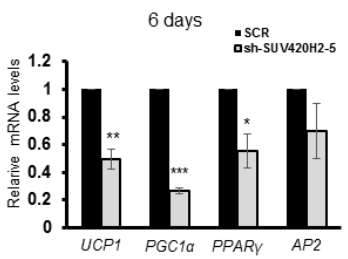

Fig. 3. Validation of proteins showing differentially expressed pattern during brown adipocyte differentiation. (A) The mRNA expressions of PRDM9, JHDM1D, MLL3 and PHF8 were investigated by real-time PCR. (B) The change in the mRNA expression of SUV42OH2 was monitored during adipocyte differentiation using both brown preadipocytes and 3T3-L1 cells (white preadipocytes). (C) The change in the protein expression of SUV420H2 was assessed during adipocyte differentiation using both brown preadipocytes and 3T3-L1 cells.

Fig. 4. The knock-down of SUV42OH2 suppresses brown adipocyte differentiation. (A) RFP expression was checked under a fluorescence microscope. (B) The knock-down of SUV42OH2 was assessed and confirmed by real-time PCR. (C) The knock-down of SUV420H2 was confirmed by a western blot analysis on day 6 using an anti-SUV420H2 antibody. (D, E) The SUV42OH2 knockdown cells were induced to differentiate, and subsequently stained with Oil-Red $\mathrm{O}$ to determine the lipid amount. (F) The expression changes of the brown adipocyte-specific genes UCP1, PCC $1 \alpha$, $P P A R \gamma$ and $a P 2$ upon the knock-down of SUV2OH2 were measured by real-time PCR. 
brown adipocyte differentiation. To clarify the role of $\mathrm{SUV} 42 \mathrm{OH} 2$ during this process, we infected immortalized brown preadipocytes with shRNA against SUV42OH2 using a retroviral expression system (shRNA-SUV420H2-RFP). A control vector containing a scrambled sequence was used as a negative control. The infected preadipocytes were isolated using a FACS sorter. Most of the cells were found to be RFP-positive under a fluorescence microscope (Fig. 4A). The knock-down of the endogenous SUV420H2 was examined by real-time PCR and western blot analysis (Figs. 4B and 4C). As shown, the shSUV420H2-5 construct appears to induce the efficient knock-down of the expression of SUV42OH2. Next, preadipocytes infected with shSUV420H2-5 were induced to differentiate into mature brown adipocytes, after which Oil-red O staining was used to measure the degree of lipid accumulation after six days of differentiation (Figs. 4D and 4E). We observed that the knock-down of $S U V 42 O H 2$ induced a significant suppression of brown adipocyte differentiation compared to that of the scrambled control. Additionally, several key adipogenic markers, i.e. UCP1, PCC1 $\alpha, P P A R \gamma$ and aP2, were significantly decreased upon the knock-down of SUV42OH2 (Fig. 4F). These results suggest that the $\mathrm{H} 4 \mathrm{~K} 20$ trimethylation by SUV420H2 at a late stage of differentiation may be an essential process during brown adipocyte differentiation.

Recently, much attention has focused on protein lysine methylation due to its central role in regulating gene expression and its close involvement in numerous key cellular processes, such as apoptosis, cell cycling, and differentiation. Adipose tissue is an essential metabolic endocrine organ that critically affects insulin sensitivity and energy homeostasis. Specifically BAT, which is mainly composed of brown adipocytes, has received a great deal of interest as a potential solution to obesity and related disorders. In relation to this, a deeper understanding of the molecular mechanisms of brown adipocyte differentiation is an essential prerequisite. In this study, we undertook a profiling analysis of the enzymes involved in lysine methylation during brown adipocyte differentiation. The several enzymes involved in lysine methylation were identified as differentially expressed proteins. Based on the results of knock-down experiments, we suggest that SUV420H2 methyltransferase may be involved in brown adipocyte differentiation.

PRDM9, a C2H2-type zinc-finger DNA-binding methyltransferase, was identified as an up-regulated enzyme during brown adipocyte differentiation. PRDM9 is a histone methyltransferase that specifically trimethylates the Lys- 4 of histone $\mathrm{H} 3$; it is essential for proper meiotic progression. H3K4 methylation is a hallmark of epigenetic transcriptional activation. Thus far, there has been no report of a relationship between PRDM9 and adipocyte differentiation. However, our profiling results suggest that PRDM9 is involved in brown adipocyte differentiation via the methylation of $\mathrm{H} 3 \mathrm{~K} 4$ or other target(s).

JHDM1D (also known as KDM7A or KIAA1718) is a lysine demethylase that is also up-regulated during brown adipocyte differentiation. This enzyme is specific to both $\mathrm{H} 3 \mathrm{~K} 9 \mathrm{me} 2$ and H3K27me2, which are important post-translational modifications associated with transcriptional silencing $(15,16)$. It was reported that JHDM1D is involved in neuronal differentiation via FGF4 and brain development $(17,18)$. In addition, the increased expression of JHDM1D occurs under starvationinduced repressed tumor growth by suppressing angiogenesis (19). Thus far, no reports have been published about a link between adipocyte differentiation and obesity. However, our results imply that JHDM1D is involved in brown adipocyte differentiation by means of transcriptional activation through the demethylation of repressive methyl markers ( $\mathrm{H} 3 \mathrm{~K} 9 \mathrm{me} 2$ and H3K27me2) (20).

MLL3 is a KMT that methylates the Lys-4 of histone $\mathrm{H} 3$ (H3K4). H3K4me is a tag for epigenetic transcriptional activation $(21,22)$. MLL3, together with MLL2, is a constituent of a large protein complex known as the ASC-2/NCOA6 complex (ASCOM), which has been shown to be a transcriptional modulator of $\beta$-globin and estrogen genes. It is also known that ASCOM plays critical roles in adipocyte differentiation as a coactivator of the key adipogenic transcriptional factors PPAR $\gamma$ and $\mathrm{C} / \mathrm{EBP} \alpha(22,23)$. In addition, there is a close relationship between $\mathrm{H} 3 \mathrm{~K} 4$ trimethylation and adipocyte differentiation. Therefore, the increased expression of MLL3 during brown adipocyte differentiation coincides well with the findings of previous reports.

In conclusion, these results of KMTs and KDMs profiling analysis, together with the further characterization of the enzymes showing differential expression patterns, should provide useful information leading to a deeper understanding of brown adipocyte differentiation.

\section{MATERIALS AND METHODS}

\section{Differentiation of immortalized brown preadipocytes}

The immortalized brown preadipocyte cell line was kindly provided by Prof. Shingo Kajimura (UCSF, USA) (24). Cells were maintained and cultured in a growth medium [high glucose Dulbecco's Modified Eagle's Medium (DMEM) containing a $1 \%$ antibiotic-antimycotic solution and $20 \%$ fetal bovine serum; Gibco-Invitrogen] at $37^{\circ} \mathrm{C}$ in a humidified atmosphere with $5 \% \mathrm{CO}_{2}$. The immortalized brown preadipocytes were induced to differentiate into mature brown adipocytes by a previously described method $(25,26)$.

\section{Oil-Red O staining}

Lipid droplets of differentiating or mature brown adipocytes were assessed by Oil-red $\mathrm{O}$ staining, as described previously $(25,26)$. For a quantification analysis, the Oil-red O staining dye was extracted and quantified, as described previously (26-30). 


\section{Quantification of KMTs and KDMs during brown adipocyte differentiation}

Total RNA was prepared by extraction from cultured cells using the QIAzol lysis reagent (Qiagen, Hilden, Germany), according to the manufacturer's instructions. First-strand complementary DNA (cDNA) was synthesized using total RNA $(2 \mu \mathrm{g})$ as a template, random primers (500 ng), and cDNA synthesis kit components (Promega) in a total volume of $25 \mu \mathrm{l}$ $(24,25)$. The targeted fragment of cDNA for each of the genes associated with adipocyte differentiation was amplified by PCR with $2 \mu \mathrm{l}$ of the reverse transcription (RT) product, 10 pmol of each primer, and a PCR premix (Nanohelix, Daejeon, Korea). In three sets of experiments, KMTs or KDMs showing significant changes in all sets were defined as differentially expressed.

\section{Analysis of gene expression}

The targeted fragment of cDNA for the brown adipocyte differentiation-associated genes was amplified by Quantitative Real-time RT-PCR (31). Primer used: SUV42OH2 (F: 5'-CGT GCTTGGAAGAAGAATGA-3'， R: 5'-GCAGTCATGGTTGATG AAGG-3'), UCP1 (F: 5'-CTTTGCCTCACTCAGGATTGG-3', R: 5'-ACTGCCACACCTCCAGTCATT-3'), PGC $1 \alpha$ (F: 5'-CCCTGC CATTGTTAAGACC-3', R: 5'-TGCTGCTGTTCCTGTTTTC-3'), PPAR $\gamma$ (F: 5'-CAAGAATACCAAAGTGCGATCAA-3', R: 5'-GA GCTGGGTCTTTTCAGAATAATA-3'), aP2 (F: 5'-TGGAAAGT CGACCACAATAAAGAG-3', R: 5'-CACCACCAGCTTGTCACC AT-3'). Gene expression levels were normalized to TBP (F: 5'-CCCCTTGTACCCTTCACCAAT-39, R: 5'-GAAGCTGCGGT ACAATTCCAG-3').

\section{SUV420H2 knock-down by shRNA using a retroviral expression system}

To knock-down the expression of $S U V 42 O H 2$ in immortalized brown preadipocytes, a retrovirus-mediated infection system was used. The gene encoding shRNA against SUV42OH2 was inserted into the multi-cloning site of the pSIREN-RetroQDsRed vector (Clontech). Subsequently, retroviruses were produced by transiently co-transfecting GP2-293 cells with a retroviral vector and the VSV-G plasmid. At $48 \mathrm{~h}$ after transfection, media containing the retroviruses were collected, filtered with $0.45-\mu \mathrm{m}$ filters, and used to infect cells in the presence of polybrene $(8 \mu \mathrm{g} / \mathrm{ml})$. Infected preadipocyte cells were selectively enriched by means of fluorescence-activated cell sorting (FACS; FACSAria cell sorter, BD Biosciences), and were then maintained in a growth medium as described previously $(25,26)$.

\section{ACKNOWLEDGEMENTS}

This work was supported by grants from KRIBB and the Research Program (2012M3A9C7050101, 2013M3A9A7046301, 2015M3A7B6027948, and 2015M3A9D7029882) of the Korea National Research Foundation.

\section{REFERENCES}

1. Park A, Kim WK and Bae K-H (2014) Distinction of white, beige and brown adipocytes derived from mesenchymal stem cells. World J Stem Cells 6, 33-42

2. Harms $M$ and Seale $P$ (2013) Brown and beige fat: development, function and therapeutic potential. Nature Med 19, 1252-1263

3. Yao X, Shan S, Zhang Y and Ying H (2011) Recent progress in the study of brown adipose tissue. Cell Biosci 1,35

4. Bae K-H, Kim WK and Lee SC (2012) Involvement of protein tyrosine phosphatases in adipogenesis: New anti-obesity targets?. BMB Rep 45, 700-706

5. Whetstine JR (2014) Methylation: a multifaceted modification - looking at transcription and beyond. Biochim Biophys Acta 1839, 1351-1352

6. Biggar KK and Li SS (2015) Non-histone protein methylation as a regulator of cellular signalling and function. Nature Rev Mol Cell Biol 16, 5-17

7. Hamamoto R, Saloura V and Nakamura Y (2015) Critical roles of non-histone protein lysine methylation in human tumorigenesis. Nature Rev Cancer 15, 110-124

8. Zhang $X$, Huang $Y$ and Shi $X$ (2015) Emerging roles of lysine methylation on non-histone proteins. Cell Mol Life Sci 72, 4257-4272

9. Okamura M, Inagaki T, Tanaka T and Sakai J (2010) Role of histone methylation and demethylation in adipogenesis and obesity. Organogenesis 6, 24-32

10. Ohno H, Shinoda K, Ohyama K, Sharp LZ and Kajimura S (2013) EHMT1 controls brown adipose cell fate and thermogenesis through the PRDM16 complex. Nature 504, 163-167

11. Del Rizzo PA and Trievel RC (2014) Molecular basis for substrate recognition by lysine methyltransferases and demethylases. Biochim Biophys Acta 1839, 1404-1415

12. Souza PP, Volkel P, Trinel D et al (2009) The histone methyltransferase SUV420H2 and heterochromatin proteins HP1 interact but show different dynamic behaviours. BMC Cell Biol 20, 41

13. Schotta G, Sengupta R, Kubicek S et al (2008) A chromatin-wide transition to $\mathrm{H} 4 \mathrm{~K} 20$ monomethylation impairs genome integrity and programmed DNA rearrangements in the mouse. Genes Dev 22, 2048-2061

14. Shinchi $Y$, Hieda M, Nishioka $Y$ et al (2015) SUV42OH2 suppresses breast cancer cell invasion through down regulation of the $\mathrm{SH} 2$ domain-containing focal adhesion protein tensin-3. Exp Cell Res 334, 90-99

15. Tachibana $M$, Sugimoto $K$, Fukushima $T$ and Shinkai $Y$ (2001) Set domain-containing protein, G9a, is a novel lysine-preferring mammalian histone methyltransferase with hyperactivity and specific selectivity to lysines 9 and 27 of histone H3. J Biol Chem 276, 25309-25317

16. Barski A, Cuddapah S, Cui K et al (2007) High-resolution profiling of histone methylations in the human genome. Cell 129, 823-837

17. Huang C, Xiang Y, Wang Y et al (2010) Dual-specificity histone demethylase KIAA1718 (KDM7A) regulates neuronal differentiation through FGF4. Cell Res 20, 
154-165

18. Tsukada Y, Ishitani T and Nakayama KI (2010) KDM7 is a dual demethylase for histone H3 Lys 9 and Lys 27 and functions in brain development. Genes Dev 24, 432-437

19. Osawa T, Muramatsu M, Wang F et al (2011) Increased expression of histone demethylase JHDM1D under nutrient starvation suppresses tumor growth via downregulating angiogenesis. Proc Natl Acad Sci U S A 108, 20725-20729

20. Yokoyama A, Okuno Y, Chikanishi T et al (2010) KIAA1718 is a histone demethylase that erases repressive histone methyl marks. Gene Cells 15, 867-873

21. Hess JL (2004) Mechanisms of transformation by MLL. Crit Rev Eukaryot Gene Exp. 14, 235-254

22. Dreijerink KM, Varier RA, van Beekum O et al (2009) The multiple endocrine neoplasia type 1 (MEN1) tumor suppressor regulates peroxisome proliferator-activated receptor gamma-dependent adipocyte differentiation. Mol Cell Biol 29, 5060-5069

23. Lee J, Saha PK, Yang Q-H et al (2008) Targeted inactivation of MLL3 histone H3-Lys-4 methyltransferase activity in the mouse reveals vital roles for MLL3 in adipogenesis. Proc Natl Acad Sci 105, 19229-19234

24. Seale P, Kajimura S, Yang W et al (2007) Transcriptional control of brown fat determination by PRDM16. Cell
Metab 6, 38-54

25. Kim WK, Oh K-J, Choi H-R et al (2015) MAP kinase phosphatase 3 inhibits brown adipocyte differentiation via regulation of ERK phosphorylation. Mol Cell Endocrinol 416, 70-76

26. Kamal AH, Kim WK, Cho K et al (2013) Investigation of adipocyte proteome during the differentiation of brown preadipocytes. J Proteomics 94, 327-336

27. Lee DS, Choi H, Han BS et al (2016) c-Jun regulates adipocyte differentiation via the KLF15-mediated mode. Biochem Biophys Res Comm 469, 552-558

28. Choi H-R, Kim WK, Park A et al (2013) Protein tyrosine phosphatase profiling studies during brown adipogenic differentiation of mouse primary brown preadipocytes. BMB Rep 46, 539-543

29. Kim EY, Kim JW, Kim WK et al (2014) Selection of aptamers for mature white adipocytes by Cell SELEX using flow cytometry. PLoS ONE 9, e97747

30. Kim HJ, Yoon BK, Park H et al (2016) Caffeine inhibits adipogenesis through modulation of mitotic clonal expression and the AKT/GSK3 pathway in 3T3-L1 adipocytes. BMB Rep 49, 111-115

31. Hu L, Su P, Li R et al (2015) Knockdown of microtubule actin crosslinking factor 1 inhibits cell proliferation in MC3T3-E1 osteoblastic cells. BMB Rep 48, 583-588 